\title{
Feuilleton.
}

\section{Berthold Kern (Berlin), Weltanschauungen und Welt-} erkenntnis. $\left.{ }^{1}\right)$

Besprochen von Priv.-Doz.Dr.phil.M. Fr ischeis en-Köhler (Berlin).

Das Werk, das der Philosophischen Fakultät der Friedrich Wilhelms-Universität zu Berlin gewidmet ist, ist aus dem Bediurfnis des Verfassers hervorgegangen, seinen vielfachen Bestrebungen um Aufrechterhaltung des alten geschichtlichen Zusammenhanges zwischen Philosophie und Naturwissenschaften, welche durch Verleihung des philosophischen FhrenDoktors die bedeutendste Anerkennung gefunden haben, einen einheitlichen Abschluß zu geben. So gibt es ebensowenig systematische Philosophie wie systematische Naturwissenschaft, sondern die systematische Anwendung beider aufeinander. Das besondere Ziel, welches das Buch verfolgt, ist in dem Titel zum Ausdruck gebracht; es will das Verhältnis der Weltanschauung zur Welterkenntnis klären und bestimmen. Damit greift es in die immer zunehmenden philosophischen Bemühungen unserer Tage um die Gewinnung einer einheitlichen Weltanschauung fördernd ein.

Ueber die Möglichkeit, auf wissenschaftlicher Grundlage eine einheitliche Weltanschauung zu errichten, gehen die Meinungen weit auseinander. Während auf der einen Seite ungestüm eine neue Weltanschauung gefordert und verkündigt wird, hat auf der andern Seite ein vorsichtiger Skeptizismus sich mehr und mehr Anerkennung gesichert, der in dem bunten Wechsel der philosophischen Standpunkte und Welterklärungen, welche die Geschichte zeigt, zwar nicht gesetzlose Anarchie, aber doch nur die Abwandlung gewisser letzter Formen von Weltbetrachtungen erblickt, die einander ausschließen, von denen aber keine das Alleinrecht auf Wahrheit beweisen kann. Aus dieser Sachlage ist das Problem entstanden, das schon seit längerer Zeit unsere besten Denker beschäftigt, wie dem Verlangen nach einer umfassenden Welttheorie, die als notwendiger Abschluß oder als Grundlage jeder Erkenntnis erscheint, bei Anerkennung der Relativität aller einzelnen Weltanschauungen Genüge zu leisten sei, wie die letzteren als Variationen, als notwendige Abwandlungen oder Einschränkungen jener zu begreifen seien. Der Verfasser gibt in dem vorliegenden Buche diesem Problem einer allgemeinen Weltanschauungslehre eine äußerst prägnante Fassung und, was mehr ist, eine, wohlbegründete, bis aufs einzelnste durchgeführte und durchdachte Auflösung.

Entscheidend für sie ist, einen überragenden Gesichtspunkt zu finden, unter welchem die synthetische Vereinigung und gegenseiti_e Abwägung der verschiedenen Anschauungsweisen eine umfassende, befriedigende und die scheinbaren Gegensätze versöhnende Welterkenntnis zu liefern vermag, in der die geschichtlichen Durchgangsstufen ihre Rechtfertigung finden und die verschiedenen Arbeitsmethoden und Denkweisen der Wissenschaften ihre sachgemäße Bewertung und ihren gegenseitigen Ausgleich erfahren. Diesen Gesichtspunkt entdeckt der Verfasser in dem Begriff der Erkenntnis überhaupt, da es auf allen Gebieten lediglich die ungenügende Aufschließung dieses Begriffes und seiner einseitigen Deutung ist, welche die Skepsis auf ihren Schultern trägt.

Was ist Erkenntnis? Erkenntnis ist mit der objektiven Wirklichkeit nicht ein und dasselbe, wie das naive Denken es sich aus-

1) Berlin 1911, A. Hirs:hwald. VII u. 459 S. 10,00 M. 
malt, sondern Erkennen heißt, die Wirklichkeit mittels unterscheidenden und vergleichenden Denkens, im wissenschaftlichen Sinne mittels analytischer Zerlegung und synthetischen Aufbaues angreifen, ergreifen, begreifen und die Ergebnisse dieses Prozesses in bestimmten Begriffen niederlegen. Die Erkenntnisbegriffe zu objektivieren, ist der Fehler des naiven Realismus, dem der landlüufige Materialismus hierin gefolgt ist; den Erkenntnisbegriffen gegenüber das Objekt zu vergessen und jene als das allein Existierende zu erklären, ist der Fehler des dogmatischen Idealismus. Im Sinne des philosophischen Kritizismus ist $\mathrm{Ob}$ jekt und Erkenntnis scharf zu scheiden; aber doch dürfen beide nicht auseinandergerissen werden. Thre Wiedervereinigung liegt fest begründet in dem Verhältnis des Teiles zum Ganzen, in der Tatsache, daß auch unsere Erkenntnis ein Teil im Ganzen der Wirklichkeit ist, daß sie von ihr abhängt und von ihr erzeugt ist, daß im erkennenden Ich das erlebende Subjekt und das erlebte Objekt zusammenfallen. Somit führt die Einsicht in den Unterschied zwischen Erkenntnis und Wirklichkeit zugleich zu der Einsicht in das Wesen dieses Unterschiedes und in die Art des Zusammenhanges zwischen Erkenntnis und Wirklichkeit.

Aber sie führt noch weiter, da aus ihr sogleich folgt, daß die Welterkenntnis einen zusammengesetzten und sehr verwickelten Prozeß erfordert, der nicht mehr zu anschaulicher, sondern zu bloßer kombinatorischer Einbeit des Gesamtinhaltes führt, der den einheitlichen Zusammenhang des ganzen Weltgeschehens nur mittels verschiedenartiger Anschauungs- und Auffassungsweisen durchdringt und zur Darstellung bringen läßt. Von diesem Gipfel aus betrachtet, gruppieren sich die einzelnen Weltanschauungen, die immer nur eine Anschauung zugrunde legen und verfolgen, zu einem harmonischen Gesamtbilde, in welchem keine von ihnen fehlen darf, sondern jede nurr richtig begrenzt und an den richtigen Platz gestellt zu werden braucht, um in ihrem Kern und in ihrer Bedeutung für das Ganze der Welterkenntnis gewürdigt zu werden. Die wichtigsten unter diesen Weltanschauungen bilden die beiden Gruppen der naturwissenschaftlichen und der idealistischen Weltanscharung.

Aber von dem höheren Standpunkte der Welterkenntnis aus zeigt sich, daß die Trennung zweier Welten als Geisteswelt und als Natur bloße Unterschiede in der Auffassungsweise sind, die zerstückeln, was gleichartig und unzerreißbar bleibt, und den Weltinhalt nicht erschöpfen. Beide Auffassungsweisen müssen sich zur Welterkenntnis ergänzen. Die verschiedenen Weltanschau,ungen sind nichts als einseitige Versuche, den Weltinhalt in einem einzigen Begriffssystem aufzufassen. Daher die Neigung zur Aufstellung prinzipieller Dogmen, die sich vor erweitertem Blick als nicht stichhaltig erweisen, oder zur gewaltsamen Ausfüllung der Lücken mit unhaltbaren Hypothesen. Aber die Erkenntnis schreitet stets darïber hinaus. Polypenartig streckt sie ihre Arme nach allen Teilen des Weltgeschehens aus, das in breitem Strome an ihr vorüberzieht, und erkennt dessen Umfang und Inhaltsfülle. Sie wächst sich aus und verzweigt sich in vorher ungeahnte Forschungsgebiete und Forschungsmethoden und gewinnt selber einen Umfang, der ihren einheitlichen Kern vielleicht vorübıı gehend vergessen läßt, aber sie besinnt sich auch wieder auf diesen und ruft nach gemeinsamer Arbeit.

Die Tragweite dieser Theorie von Welterkenntnis und Weltanschauung, die der Verfasser in der bei ihm rühmlich bekannten lichtvollen Darstellung entwickelt, festzustellen, wird Aufgabe der philosophischen Fachkritik sein. Aber da das Buch sich nicht nur an die philosophischen Fachgelehrten, sondern an alle die wendet, denen die Frage nach einer einheitlichen Weltanschauung und Welterkenntnis am Herzen liegt, wird es das Ziel des Verfassers, den fruchtbaren Wechselverkehr von Naturwissenschaft mit der Philosophie zu fördern, gewiß erreichen. 